\title{
In situ hydrogenation of the Zintl phase SrGe
}

\author{
Henry Auer, ${ }^{\dagger}$ Dirk Wallacher, ${ }^{\ddagger}$ Thomas Christian Hansen, ${ }^{\top}$ and Holger
}

\author{
Kohlmann*,†
}

$\dagger$ Leipzig University, Department of Inorganic Chemistry, Johannisalle 29, 04103 Leipzig, Germany

$\ddagger$ Helmholtz Zentrum Berlin für Materialien und Energie, Hahn-Meitner-Platz 1, 14109

$$
\text { Berlin, Germany }
$$

IInstitut Laue-Langevin, 71 Avenue des Martyrs, CS 20156, 38042 Grenoble cedex 9, France

E-mail: holger.kohlmann@uni-leipzig.de

Phone: +49 341/97-36201. Fax: +49 341/97-36199 


\begin{abstract}
Hydrides (deuterides) of CrB-type Zintl phases AeTt $(\mathrm{Ae}=$ alkaline earth; $\mathrm{Tt}=$ tetrel) show interesting bonding properties with novel polyanions. In $\mathrm{SrGeD}_{4 / 3-\mathrm{x}}(\gamma-$ phase) three zig-zag chains of Ge atoms are condensed and terminated by covalently bound deuterium atoms. A combination of in situ techniques (thermal analysis, synchrotron and neutron powder diffraction) revealed the existence of two further hydride (deuteride) phases with lower $\mathrm{H}(\mathrm{D})$ content (called $\alpha$ - and $\beta$-phases). Both are structurally related to the parent Zintl phase SrGe and to the ZrNiH-structure type containing variable amounts of hydrogen (deuterium) in $\mathrm{Sr}_{4}$ tetrahedra. For $\alpha-\mathrm{SrGeD}_{\mathrm{y}}$ the highest $\mathrm{D}$ content $\mathrm{y}=0.29$ was found at $575(2) \mathrm{K}$ under 5.0(1) MPa deuterium pressure, $\beta-\mathrm{SrGeD}_{\mathrm{y}}$ shows a homogeneity range of $0.47<\mathrm{y}<0.63$. Upon decomposition of $\mathrm{SrGeD}_{4 / 3-\mathrm{x}}\left(\gamma-\mathrm{SrGeD}_{\mathrm{y}}\right)$ tetrahedral $\mathrm{Sr}_{4}$-voids stay filled while the germanium bound D4-site looses deuterium. When reaching the lower D content limit, $\mathrm{SrGeD}_{4 / 3-\mathrm{x}}$ ( $\gamma$-phase), $0.10<\mathrm{x}<0.17$ decomposes to the $\beta$-phase. All three hydrides (deuterides) of SrGe show variable $\mathrm{H}(\mathrm{D})$ content.
\end{abstract}

\title{
Introduction
}

Zintl phases build a bridge between solid state and molecular chemistry. These polar intermetallic compounds exhibit polyanionic partial structures that resemble molecular analogues. Structures of Zintl phases can be explained by a simple concept where the less electronegative partner formally transfers all its valence electrons to the more electronegative one. ${ }^{1-7}$ As expected this concept lacks to describe the true electronic situation, e.g. CaSi crystallizes in CrB-structure type and is a textbook example of a Zintl phase, where the formally $\mathrm{Si}^{2-}$ forms a zig-zag chain-like partial structure that is coordinated by $\mathrm{Ca}^{2+}$ cations. In contrast to this ionic picture that suggests semi-conducting properties all the isotypic compounds $\operatorname{AeTt}(\mathrm{Ae}=\mathrm{Ca}-\mathrm{Ba}, \mathrm{Tt}=\mathrm{Si}, \mathrm{Ge})$ are poor metals, ${ }^{8,9}$ i.e., the Zintl-Klemm formalism is a very useful crystal chemistry concept, which, however often lacks a proper description of the 
electronic structure. DFT and experimental charge density studies could show significant covalent contribution for the Ca-Si bonding. ${ }^{10}$

Introducing hydrogen (deuterium) into these systems complicates this picture even further. There are several hydride (deuteride) phases that derive from CrB-type structures like $\mathrm{CaSiH}_{1.2},{ }^{11-14} \mathrm{SrSiH}_{1.6},{ }^{14} \mathrm{BaSiH}_{2-\mathrm{x}},{ }^{14,15} \mathrm{SrGeH}_{4 / 3-\mathrm{x}},{ }^{15} \mathrm{BaSnH}_{4 / 3-\mathrm{x}},{ }^{15} \mathrm{NdGaH}_{1.66},{ }^{16} \mathrm{GdGaH}_{\mathrm{y}}{ }^{17}$ Common features in these phases are salt like layers of hydrogen (deuterium) filled $\mathrm{M}_{4^{-}}$ tetrahedra ( $\mathrm{M}=\mathrm{Ca}-\mathrm{Ba}, \mathrm{Nd}, \mathrm{Gd})$ as well as hydrogen (deuterium) positions that coordinate the polyanionic chain. For $\mathrm{BaSiD}_{2-\mathrm{x}}, \mathrm{SrGeD}_{4 / 3-\mathrm{x}}$ and $\mathrm{BaSnD}_{4 / 3-\mathrm{x}}$ we could show that these positions correspond to covalent $\mathrm{Tt}-\mathrm{H}(\mathrm{D})(\mathrm{Tt}=\mathrm{Si}-\mathrm{Sn})$ bonds using neutron diffraction, solid state NMR spectroscopy as well as DFT calculations. ${ }^{15}$ In view of the variety of different related crystal structures it seemed promising to follow the uptake of hydrogen (deuterium) by time-resolved studies. In situ diffraction techniques in general ${ }^{18,19}$ can help to probe phases that occur during a reaction. Especially intermediate products that cannot be quenched are accessible. Moreover composition changes can be followed to estimate homogeneity ranges for different phases.

In this contribution we present in situ powder neutron diffraction (in situ PND) data on reactions in the $\mathrm{SrGe}-\mathrm{H}_{2}\left(\mathrm{D}_{2}\right)$ system. The findings are correlated to a thermal analysis of SrGe under different hydrogen pressures as well as in situ powder synchrotron diffraction. In situ investigations on $\mathrm{SrGe}$ shows, in addition to $\mathrm{SrGeD}_{4 / 3-\mathrm{x}}\left(\right.$ called $\gamma-\mathrm{SrGeD}_{\mathrm{y}}$ ), the existence of two hydrogen (deuterium) poorer phases ( $\alpha$ - and $\beta$-phase), that both are structurally related to the $\mathrm{ZrNiH}-$ structure type ${ }^{20}$. They exhibit a large homogeneity range of their hydrogen (deuterium) content. The same applies to $\operatorname{SrGeD}_{4 / 3-\mathrm{x}}$ with a large homogeneity range for the hydrogen (deuterium) position covalently bound to germanium. 


\section{Experimental}

\section{Synthesis}

All handlings were done in an argon filled glovebox. Oxygen and moisture level were kept below 1 ppm. SrGe and $\operatorname{SrGeD}_{4 / 3-\mathrm{x}}\left(\gamma-\mathrm{SrGeD}_{\mathrm{y}}\right)$ were prepared from the elements (Sr: chemPUR, 99\%, packed under oil and washed in hexane; Ge: chemPUR, 99.9999\%) and subsequent deuteration $\left(\mathrm{D}_{2}\right.$ : Air Liquide, $99.8 \%$ isotope purity) of the Zintl phase as described before. ${ }^{15}$

\section{Thermal Analysis}

Differential scanning calorimetry (DSC) was performed with a Q1000 DSC (TA Instruments) equipped with a gas pressure chamber. DSC experiments were done under several hydrogen pressures in the region of 2 to $5 \mathrm{MPa}$ to follow the hydrogenation reaction. Aluminum crucibles were filled with about $15 \mathrm{mg}$ SrGe and crimped within a glovebox. The sample was placed in the pressure chamber of the DSC, which was subsequently flushed with hydrogen and afterwards filled to the desired hydrogen pressure. Samples were heated at a rate of $10 \mathrm{~K} \cdot \mathrm{min}^{-1}$ to a maximum temperature of $700 \mathrm{~K}$. The temperature was usually held for 10 minutes. In subsequent runs lower maximum temperatures were used depending on the occurring signals. The heating was then stopped right after a reaction step, the temperature held there for 10 minutes before cooling down to room temperature.

\section{Structural Characterization}

Powder Neutron Diffraction (PND) was done at the Institut Laue Langevin (ILL), Grenoble, France at the high-intensity two-axis diffractometer D20. ${ }^{21}$ Measurements were done at a take off angle of $120^{\circ}$ and a wavelength of $\lambda=1.86832(7) \AA$ which was calibrated using a silicon NIST640b standard sample in a $5 \mathrm{~mm}$ vanadium container. Ex situ measurements were conducted in indium sealed $6 \mathrm{~mm}$ vanadium containers. In situ experiments 
were done in (leuco-)sapphire single crystal cells with $6 \mathrm{~mm}$ inner diameter, which were connected to a gas supply system (for more details see Kohlmann et al. ${ }^{22}$ ). For the in situ investigation the sapphire cell filled with $\mathrm{SrGe}$ or $\operatorname{SrGeD}_{4 / 3-\mathrm{x}}\left(\gamma-\mathrm{SrGeD}_{\mathrm{y}}\right)$ was pressurized with 5.0(1) $\mathrm{MPa} \mathrm{D}_{2}$ (Air Liquide, 99.8\% isotope purity) at ambient temperature. Heating up to a maximum temperature of $623 \mathrm{~K}$ was realized using two laser beams. Data were collected with 2 min time resolution. For sequential Rietveld refinement a summation over 5 data sets was done giving a time resolution of $10 \mathrm{~min}$. Sequential data are presented with error bars corresponding to 3 e.s.u. as obtained from Rietveld refinement. For more clarity 2D-diffraction patterns are truncated to a small angular range. The total measured angular range is presented in Fig. S2 (SrGe) and Fig. S3 $\left(\gamma-\mathrm{SrGeD}_{\mathrm{y}}\right)$.

All data sets obtained at the ILL D20 instrument are presented with an additional label according internal raw data labeling (NUMOR-labeling). Data refer to proposal 5-22-734. ${ }^{23}$

Ex situ PND was also performed at the neutron source BER II at Helmholtz-Zentrum Berlin (HZB), Germany at the high resolution powder diffractometer E9 (FIREPOD). ${ }^{24}$ Measurements were done at a wavelength of $1.7972(2) \AA$ in a copper sealed $6 \mathrm{~mm}$ vanadium container.

In situ Powder Synchrotron Diffraction (in situ-PSD) was realized at BESSY II, Helmholtz-Zentrum Berlin (HZB), Germany at beamline KMC-2. ${ }^{25}$ A novel in situ setup for diffraction under hydrogen pressure and elevated temperatures was used, which allows a qualitative analysis of the reactions. Data do not allow Rietveld refinement yet due to static samples yielding poor crystallite statistics. Details will be published elsewhere.

Rietveld refinement was done using FULLPROF. ${ }^{26,27}$ In situ data sets were evaluated in sequential refinements. If a phase showed a fraction less than $10 \%$ the atomic structure was kept fixed. When lattice parameters of such a phase diverged or showed unreasonable behavior the phase was removed from the refinement. Profile parameters were constrained to be the same for all phases. Structure pictures were prepared by VESTA. ${ }^{28,29}$ Structural 
data were normalized using STRUCTURE TIDY ${ }^{30}$ as implemented in VESTA.

\section{Results and Discussion}

\section{Structures of $\alpha$ - and $\beta-\mathrm{SrGeD}_{\mathbf{y}}$}

The thermal behavior of $\gamma-\mathrm{SrGeH}_{\mathrm{y}}\left(\mathrm{SrGeH}_{4 / 3-\mathrm{x}}\right)$ suggested the existence of two hydrogen poorer phases, which will be called $\alpha$ - and $\beta-\mathrm{SrGeH}_{\mathrm{y}}$. Structural studies reveal that the Zintl phase SrGe and the hydrogen (deuterium) richest phase $\gamma-\mathrm{SrGeD}_{\mathrm{y}}{ }^{15}$ can be considered as compositional and structural boundaries for these phases. They have in common main structural features as tetrahedral $\mathrm{Sr}_{4}$-voids and Ge-Ge zig-zag chains. While $\gamma-\mathrm{SrGeD}_{\mathrm{y}}$ has filled tetrahedral voids and partially connected Ge-Ge-chains, the voids are empty and the chains are well separated for SrGe. This suggests, that the intermediate phases may either show features of the parent Zintl phase or the hydrogen rich hydride (deuteride). Using in situ neutron diffraction data, two new phases, $\alpha$ - and $\beta-\operatorname{SrGeD}_{\mathrm{y}}$, could be indexed in the orthorhombic crystal system. In regard to the axes-system of SrGe a systematic contraction in $a$-direction and an elongation in $b$-direction can be seen with increasing deuterium content. The $c$ axis only slightly contracts.

Table 1: Structural data and selected distances $(<3 \AA)$ of $\alpha-\mathrm{SrGeD}_{\mathrm{y}}, \mathrm{y}=0.204(4)$ at 618(2) K, 5.0(1) MPa D pressure. Space group Cmcm (No.63), $a=4.7659(5) \AA, b=$ $11.8280(14) \AA, c=4.1542(5) \AA$.

\begin{tabular}{ccccccc} 
Atom & Wyckoff & $\mathrm{x}$ & $\mathrm{y}$ & $\mathrm{z}$ & $\mathrm{B}_{\text {iso }} / \AA^{2}$ & $\mathrm{SOF}$ \\
$\mathrm{Sr}$ & $4 c$ & 0 & $0.3568(2)$ & $1 / 4$ & $4.82(17)$ & \\
$\mathrm{Ge}$ & $4 c$ & 0 & $0.0679(3)$ & $1 / 4$ & $2.66(13)$ & \\
$\mathrm{D}$ & $4 c$ & 0 & $0.7509(11)$ & $1 / 4$ & $2.1(6)$ & $0.204(4)$ \\
\hline \hline Atom 1 & Atom 2 & $\mathrm{~d} / \AA$ & \\
$\mathrm{Sr}$ & $\mathrm{D}$ & $2.437(8)-2.692(7)$ & \\
$\mathrm{Ge}$ & $\mathrm{Ge}$ & $2.626(3)$ & \\
$\mathrm{Ge}$ & $\mathrm{D}$ & & $2.985(11)$ &
\end{tabular}




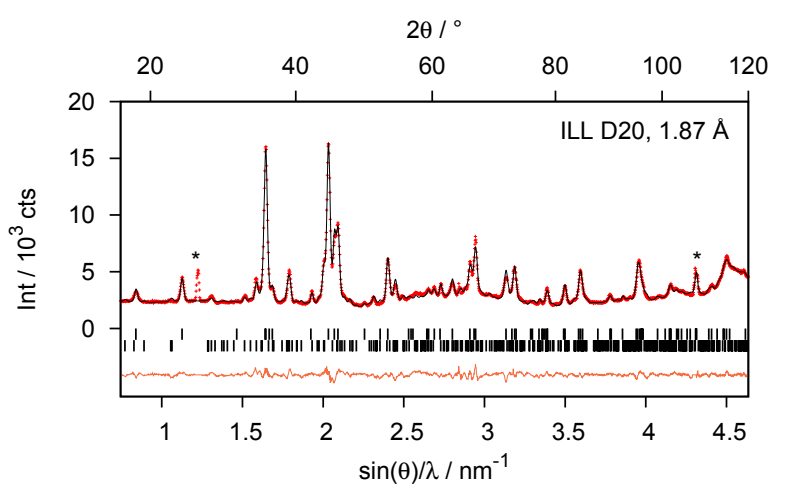

Figure 1: Rietveld refinement of the crystal structure of $\alpha-\mathrm{SrGeD}_{\mathrm{y}}(\mathrm{y}=0.204(4), \mathrm{Cmcm}$, No. $63, a=4.7659(5) \AA, b=11.8280(14) \AA, c=4.1542(5) \AA)$ based on in situ PND data at 618(2) K and 5.0(1) MPa $\mathrm{D}_{2}$. A summation was done from numor 926991 to $927020,{ }^{23}$ with an overall measuring time of $60 \mathrm{~min}$. The additional phase is $\gamma-\mathrm{SrGeD}_{\mathrm{y}}(\approx 20 \%$, bottom marker); $(*)$ denotes reflections of the sapphire cell. Background corrected reliability factors are: $\mathrm{R}_{\mathrm{p}}=13.6 \%, \mathrm{R}_{\mathrm{wp}}=13.0 \%, \mathrm{~S}=2.45$.

$\alpha-\mathrm{SrGeD}_{\mathrm{y}}$ : The structure of $\alpha-\mathrm{SrGeD}_{\mathrm{y}}$ can be described in orthorhombic $\mathrm{Cmcm}$ (No. 63) symmetry. Lattice parameter $a$ is contracted while $b$ is elongated in regard to the Zintl phase. According to a difference Fourier map there is only one deuterium site in the center of the $\mathrm{Sr}_{4}$ tetrahedral voids, which could be confirmed by a Rietveld refinement, based on the in situ collected data at 5.0(1) MPa $\mathrm{D}_{2}$-pressure and 618(2) $\mathrm{K}$ as shown in Fig. 1. This phase has the lowest hydrogen content which was refined to $y=0.204(4)$. Structural data are summarized in Tab. 1.

The structure looks quite similar to the parent Zintl phase SrGe (Fig. 2 (a/b)), but has a partial occupation of tetrahedral $\mathrm{Sr}_{4}$-voids by deuterium of $20.4(4) \%$ (Fig. 2 (c/d)). $\alpha$ $\mathrm{SrGeD}_{\mathrm{y}}$ can be classified as an interstitial hydride (deuteride) according to Häussermann. ${ }^{31}$ Applying an extended Zintl concept that includes hydrogen (deuterium) it can be considered as $\mathrm{Sr}^{2+}\left(\mathrm{H}^{-}\right)_{0.2} \mathrm{Ge}^{1.8-}$. Although the polyanion is formally oxidized, it keeps the structure we know from the hydrogen (deuterium) free Zintl phase.

As Fig. 2 (d) shows, the germanium chains stay parallel and well separated. The distance $\mathrm{d}(\mathrm{Ge}-\mathrm{Ge})=2.626(3) \AA$ at $618(2) \mathrm{K}$ is slightly shorter than in the hydrogen free Zintl phase $(\mathrm{d}(\mathrm{Ge}-\mathrm{Ge})=2.6462(16) \AA)$ at ambient temperature. Assuming a normal temperature effect, 
the bond length decreases upon hydrogenation. The Zintl phases themselves are poor metals. For the silicides this is explained in detail by $\pi$-bonding in the zig-zag chains ${ }^{9,10,32}$ due to a partial depopulation of $\pi^{*}$-bands. The reduced electron count at the polyanion in the hydride (deuteride) strengthens this effect further, therefore the decreased bond length can be correlated with increased $\pi$-bonding. The Ge-Ge-Ge angle does not change and is $104.57(18)^{\circ}\left(\right.$ SrGe: $\left.104.48(15)^{\circ}\right)$.
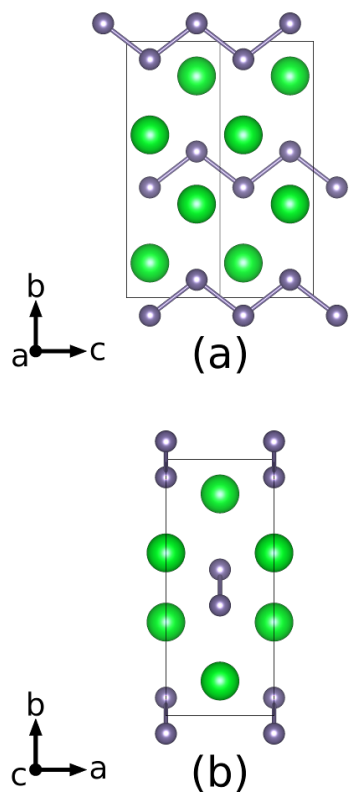

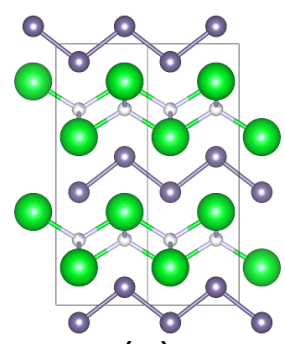

(c)

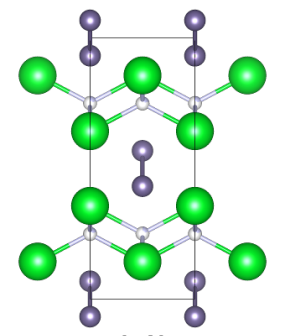

(d)
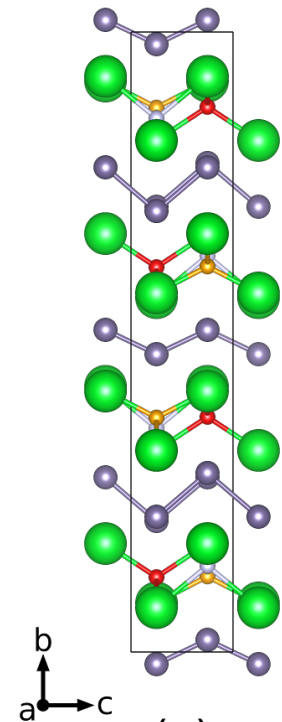

(e)

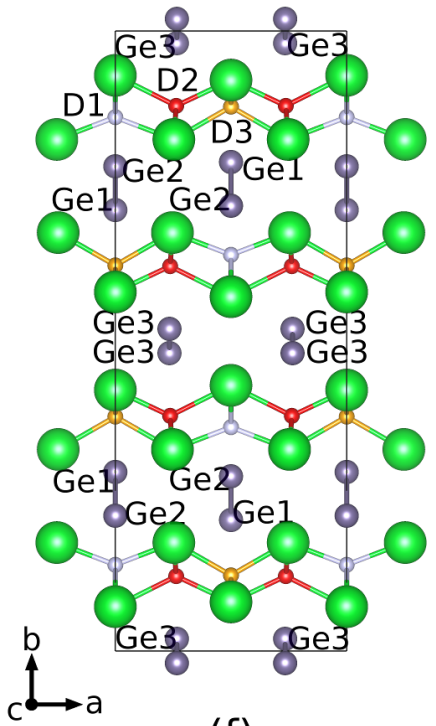

(f)

Figure 2: Different projections of the crystal structures of the Zintl phase SrGe (a/b), $\alpha$ (c/d) and $\beta-\mathrm{SrGeD}_{\mathrm{y}}(\mathrm{e} / \mathrm{f})$. Tetrahedral $\mathrm{Sr}_{4}$-voids of $\alpha$ - and $\beta-\mathrm{SrGeD}_{\mathrm{y}}$ are partially filled with deuterium. Large spheres: Sr; Medium spheres: Ge; Small spheres D.

$\beta$-SrGeD $\mathbf{y}$ : The structure is more complex than the $\alpha$ - and $\gamma$-phase. In a first approach it can be described in the basis structure of the Zintl phase with deuterium occupying tetrahedral $\mathrm{Sr}_{4}$ voids again.

Fig. 3 shows the Rietveld plot. A superstructure reflection at low angles is marked and corresponds to a $2 \times 2$ super cell in crystallographic $a$ - and $b$-direction. See also Fig. S5 for corresponding 2D-diffraction data. Rietveld refinement was done on the in situ data at 5.0(1) $\mathrm{MPa} \mathrm{D}_{2}$-pressure and 538(2) K. Due to the limited quality and the occurrence of 
the other phases a structure solution was difficult. Thus, considering the superstructure, a high symmetry model in $\mathrm{Cmcm}$ (No. 63) is suggested (Tab. 2/Fig. 2 (e/f)). The deuterium content could be refined to give the chemical formula $\beta-\mathrm{SrGeD}_{\mathrm{y}}, \mathrm{y}=0.53(4)$, which implies a half filling of the tetrahedral voids. The proposed model shows a preferred filling but allows no total ordering due to limited degrees of freedom. Different low symmetry models have been tested, but none gave a much better refinement result than the model as shown in Fig. 2 (e/f). From a Zintl point of view this phase can be considered as $\mathrm{Sr}^{2+}\left(\mathrm{H}^{-}\right)_{0.53} \mathrm{Ge}^{1 \cdot 47-} \approx$ $\mathrm{Sr}^{2+}\left(\mathrm{H}^{-}\right)_{0.5}\left(\mathrm{Ge}^{2-}\right)_{0.5}\left(\mathrm{Ge}^{-}\right)_{0.5}$.

The proposed model shows two different types of Ge zig-zag chains with d $(\mathrm{Ge} 1-\mathrm{Ge} 2)=$ $2.71(3) \AA$ and $\mathrm{d}(\mathrm{Ge} 3-\mathrm{Ge} 3)=2.280(11) \AA$ at $538(2) \mathrm{K}$. The former is not significantly different form the value of the hydrogen (deuterium) free Zintl phase $(\mathrm{d}(\mathrm{Ge}-\mathrm{Ge})=2.6462(16) \AA)$ and therefore corresponds to a single bond. The value $\mathrm{d}(\mathrm{Ge} 3-\mathrm{Ge} 3)$ is much smaller and therefore might be dominated by additional $\pi$-bonding. The corresponding Ge3-Ge3-Ge3 angle is $129.1(15)^{\circ}$ compared to the chain angle Ge1-Ge2-Ge1 of the long chain with $98.9(6)^{\circ}$.

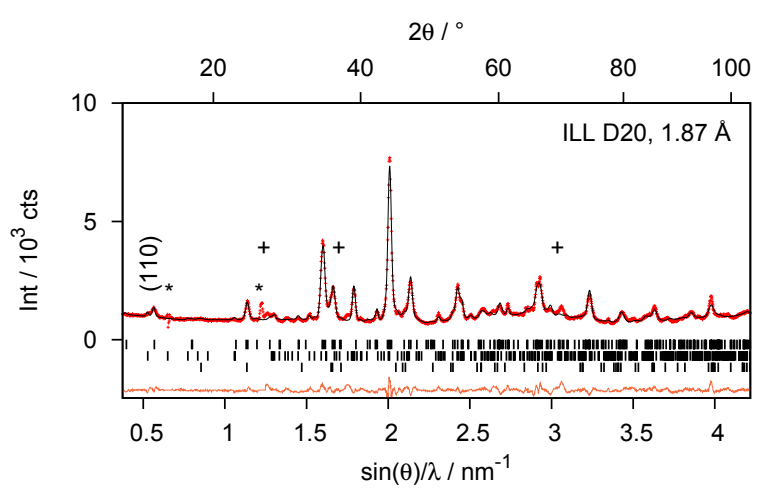

Figure 3: Rietveld refinement of the crystal structure of $\beta-\mathrm{SrGeD}_{\mathrm{y}}$ ( $\mathrm{y}=0.53(4), \mathrm{Cmcm}$, No. 63, $a=9.3368(19) \AA, b=25.001(6) \AA, c=4.1164(8) \AA$, ) based on in situ PND data at $538(2) \mathrm{K}$ and 5.0(1) $\mathrm{MPa} \mathrm{D}_{2}$. A summation was done from numor 927072 to $927082,{ }^{23}$ with an overall measuring time of $22 \mathrm{~min}$. Additional phases are $\gamma-\mathrm{SrGeD}_{\mathrm{y}}(\approx 25 \%$, middle marker $), \alpha-\operatorname{SrGeD}_{\mathrm{y}}(\approx 8 \%$, bottom marker $) ;(*)$ denotes a detector deficiency and reflections of the sapphire cell. $(+)$ denotes the largest misfits. Background corrected reliability factors are: $\mathrm{R}_{\mathrm{p}}=20.2 \%, \mathrm{R}_{\mathrm{wp}}=18.6 \%, \mathrm{~S}=2.17$. 
Table 2: Structural data and selected distances $(<3 \AA)$ of $\beta-\mathrm{SrGeD}_{\mathrm{y}}, \mathrm{y}=0.53(4)$, at $538(2) \mathrm{K}, 5.0(1) \mathrm{MPa} \mathrm{D}_{2}$ pressure. Space group Cmcm (No.63), $a=9.3368(19) \AA, b=$ 25.001(6) $\AA, c=4.1164(8) \AA$. Debye-Waller-factors were constrained for like atoms.

\begin{tabular}{|c|c|c|c|c|c|c|}
\hline Atom & Wyckoff & $\mathrm{x}$ & $\mathrm{y}$ & $\mathrm{z}$ & $\mathrm{B}_{\text {iso }} / \AA^{2}$ & $\mathrm{SOF}$ \\
\hline Sr1 & $4 c$ & 0 & $0.5792(11)$ & $1 / 4$ & $4.9(2)$ & \\
\hline Sr2 & $4 c$ & 0 & $0.0716(13)$ & $1 / 4$ & 4.9 & \\
\hline Sr3 & $8 g$ & $0.247(3)$ & $0.3250(7)$ & $1 / 4$ & 4.9 & \\
\hline Ge1 & $4 c$ & 0 & $0.7112(12)$ & $1 / 4$ & $6.7(3)$ & \\
\hline Ge2 & $4 c$ & 0 & $0.2183(15)$ & $1 / 4$ & 6.7 & \\
\hline Ge3 & $8 g$ & $0.234(2)$ & $0.4804(7)$ & $1 / 4$ & 6.7 & \\
\hline D1 & $4 c$ & 0 & $0.860(4)$ & $1 / 4$ & $2.1(4)$ & $0.24(4)$ \\
\hline D2 & $4 c$ & 0 & $0.3858(15)$ & $1 / 4$ & 2.1 & $0.65(4)$ \\
\hline D3 & $8 g$ & $0.263(5)$ & $0.1206(8)$ & $1 / 4$ & 2.1 & $0.61(3)$ \\
\hline$\overline{\text { Atom } 1}$ & Atom 2 & \multicolumn{5}{|c|}{$\overline{\mathrm{d} / \AA}$} \\
\hline $\mathrm{Sr}$ & $\mathrm{D}$ & \multicolumn{5}{|c|}{$2.237(18)-2.75(5)$} \\
\hline Ge1 & Ge2 & \multicolumn{5}{|c|}{$2.71(3)$} \\
\hline Ge3 & Ge3 & \multicolumn{5}{|c|}{$2.280(11)$} \\
\hline $\mathrm{Ge}$ & D & \multicolumn{5}{|c|}{$2.86(7)$ and $>3$} \\
\hline
\end{tabular}

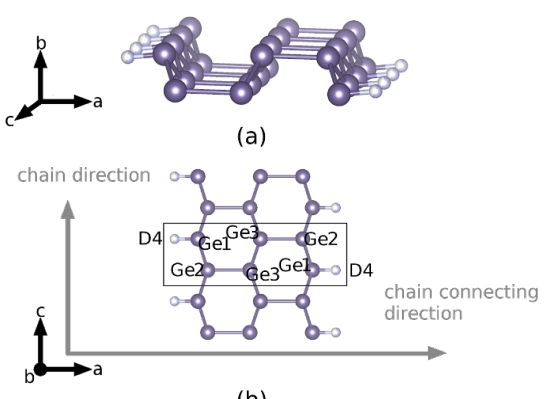

(b)

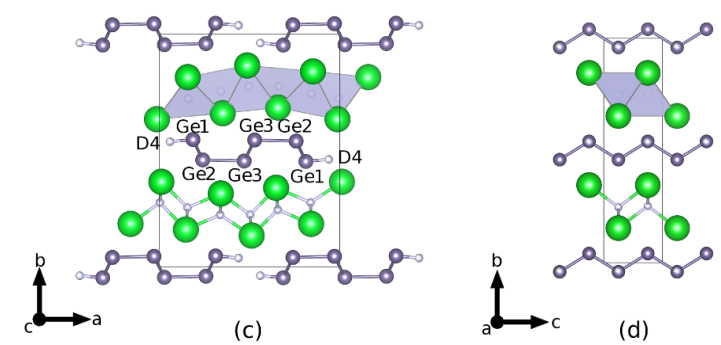

Figure 4: Polyanionic partial structure $(\mathrm{a} / \mathrm{b})$ and crystal structure $(\mathrm{c} / \mathrm{d})$ of $\gamma-\mathrm{SrGeD}_{\mathrm{y}}$ following [ 15]. Large spheres: Sr; Medium spheres: Ge; Small spheres D. 
Table 3: Lattice parameters and site occupancy factor (SOF) of the germanium bound D4 atom for $\gamma-\mathrm{SrGeD}_{\mathrm{y}}$ right after the preparation, during the in situ experiment and after 70 days under inert atmosphere. SOF of D1-D3 (tetrahedral voids) are not affected. For complete structural data see supplementary information (Tab. S1).

\begin{tabular}{cccccc} 
& 1 & 2 & 3 & 4 & 5 \\
institute $^{\text {a }}$ & BER II & ILL $^{15}$ & ILL & ILL & ILL \\
container & vanadium & vanadium & sapphire cell & sapphire cell & sapphire cell \\
& & & $0.3(1) \mathrm{MPa} \mathrm{D}_{2}$ & $5.0(1) \mathrm{MPa} \mathrm{D}_{2}$ & $5.0(1) \mathrm{MPa} \mathrm{D}_{2}$ \\
& & & $298(2) \mathrm{K}$ & $503(2) \mathrm{K}$ & $313(4) \mathrm{K}$ \\
\hline time after & & & during & after \\
preparation & +3 days & +70 days & +70 days & in situ & in situ \\
\hline Space group & Pbnm & Pbnm & Pbnm & Pbnm & Pbnm \\
& $($ No. 62$)$ & $($ No. 62$)$ & $($ No. 62$)$ & $($ No. 62) & (No. 62) \\
\hline$a / \AA$ & $11.8176(10)$ & $11.9113(9)$ & $11.9028(11)$ & $11.9435(10)$ & $11.8283(9)$ \\
$b / \AA$ & $15.3772(14)$ & $15.2692(11)$ & $15.2807(13)$ & $15.3252(13)$ & $15.3534(11)$ \\
$c / \AA$ & $4.0603(3)$ & $4.0600(2)$ & $4.0607(3)$ & $4.0728(3)$ & $4.0617(2)$ \\
\hline $\mathrm{V} / \AA^{3}$ & $737.85(18)$ & $738.41(14)$ & $738.57(19)$ & $745.47(18)$ & $737.62(15)$ \\
$\mathrm{SOF}(\mathrm{D} 4)$ & $0.684(10)$ & $0.592(8)$ & $0.616(12)$ & $0.642(12)$ & $0.672(10)$
\end{tabular}

${ }^{a}$ BER II - Helmholtz-Zentrum Berlin, Germany; ILL - Institut Laue Langevin, France

\section{Long term stability of $\gamma-\mathrm{SrGeD}_{\mathrm{y}}$}

The structure of $\gamma$-SrGeD y was presented elsewhere and discussed in detail. ${ }^{15}$ It shows Pbnm (No 62) symmetry. (A nonstandard setting is used, thus, the axes do not need to be rotated in regard to the Zintl phase SrGe. Standard setting is Pnma; $a^{\prime}=b, b^{\prime}=c, c^{\prime}=a$.) In contrast to the other phases $\gamma-\mathrm{SrGeD}_{\mathrm{y}}$ has completely filled tetrahedral $\mathrm{Sr}_{4}$-voids. The Ge-Ge chains are tilted and two thirds of the germanium atoms form an additional Ge-Ge bond. The last third of the germanium atoms is hydrogen (deuterium) terminated (site D4) or neighboring a void since the hydrogen (deuterium) site D4 is underoccupied.

Lattice metrics as well as deuterium content at the chain terminating position D4 change over time as shown by PND (Tab. 3, for complete structural data see Tab. S1). Over 70 days a hydrogen (deuterium) release at site D4 from $68 \%$ to $60 \%$ occupation can be seen. After the in situ experiment the D4-site has a comparable filling as the freshly prepared sample. Following the general trend, lattice parameter $a$ shrinks while $b$ gets larger during the hydrogen (deuterium) uptake. In contrast the overall volume effect is negative. Rietveld 
refinement plots for different data sets can be found in the supporting information file Fig. S1.

\section{Overview of the hydrogenation of SrGe followed in situ}

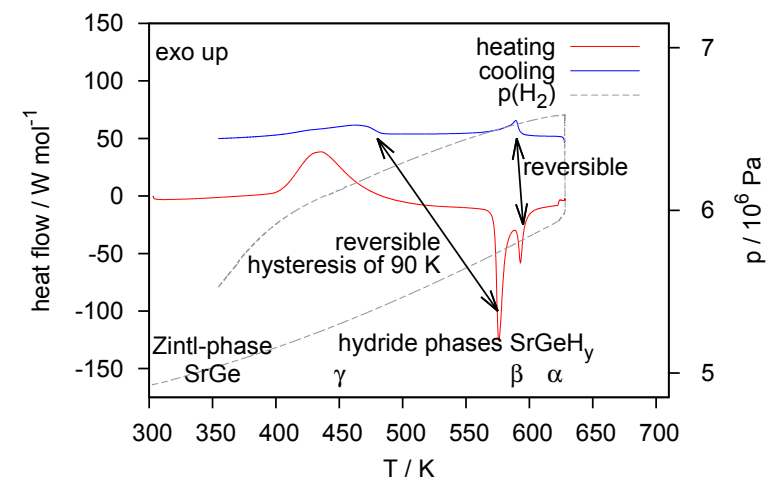

Figure 5: Hydrogen pressure DSC of the hydrogenation reaction of SrGe. Further heating (not shown) leads to a slow and irreversible decomposition resulting in $\mathrm{SrH}_{2}$ and $\mathrm{SrGe}_{2}$. This last step did not give a proper DSC signal.

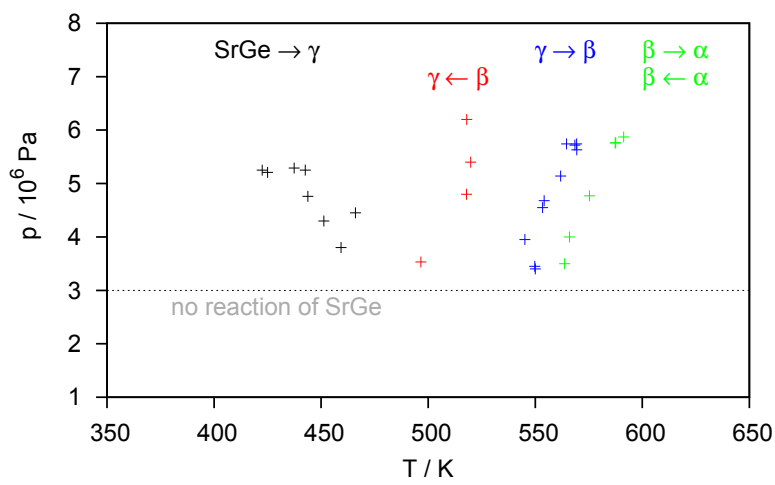

Figure 6: Onset temperatures for the different reaction steps in DSC experiments at various hydrogen gas pressures. The onset of the formation of $\gamma-\mathrm{SrGeH}_{\mathrm{y}}$ from $\mathrm{SrGe}$ is not well defined and varies for different experiments. Below $3 \mathrm{MPa}$ no reaction of SrGe was obtained.

The hydrogenation of the Zintl phase SrGe was investigated under in situ conditions. Hydrogen pressure DSC shows three prominent signals. Fig. 5 shows the heat flow during the reaction at $5 \mathrm{MPa} \mathrm{H}_{2}$ starting pressure. At $405 \mathrm{~K}$ a first strong exothermic reaction starts. The product of this step can be characterized ex situ, which has been published elsewhere as $\operatorname{SrGeD}_{4 / 3-\mathrm{x}}\left(\gamma-\mathrm{SrGeD}_{\mathrm{y}}\right) .{ }^{15}$ Afterwards two endothermic reactions follow at $570 \mathrm{~K}$ and $590 \mathrm{~K}$, respectively. Both of these steps are reversible and none of the products can be 
quenched for ex situ characterization yet. While the second endothermic step is reversible with negligible hysteresis, the first one shows a hysteresis of $90 \mathrm{~K}$. Running the reaction under $5 \mathrm{MPa} \mathrm{H}_{2}$ pressure at temperatures above $700 \mathrm{~K}$ we get the formation of $\mathrm{SrH}_{2}$ and of the germanium richer Zintl phase $\mathrm{SrGe}_{2}$. The DSC measurements were done several times at different pressure conditions. Fig. 6 shows the pressure dependency of the different reaction steps. The exothermic formation step of $\gamma-\mathrm{SrGeH}_{\mathrm{y}}$ shifts to higher temperatures on lowering the pressure. On the other hand the two subsequent endothermic steps shift to lower temperatures indicating a hydrogen loss. Thus, the hydrogen poorer phases $\beta$ - and $\alpha-\mathrm{SrGeH}_{\mathrm{y}}$ are subsequently formed. The results of the precharacterization using a hydrogen
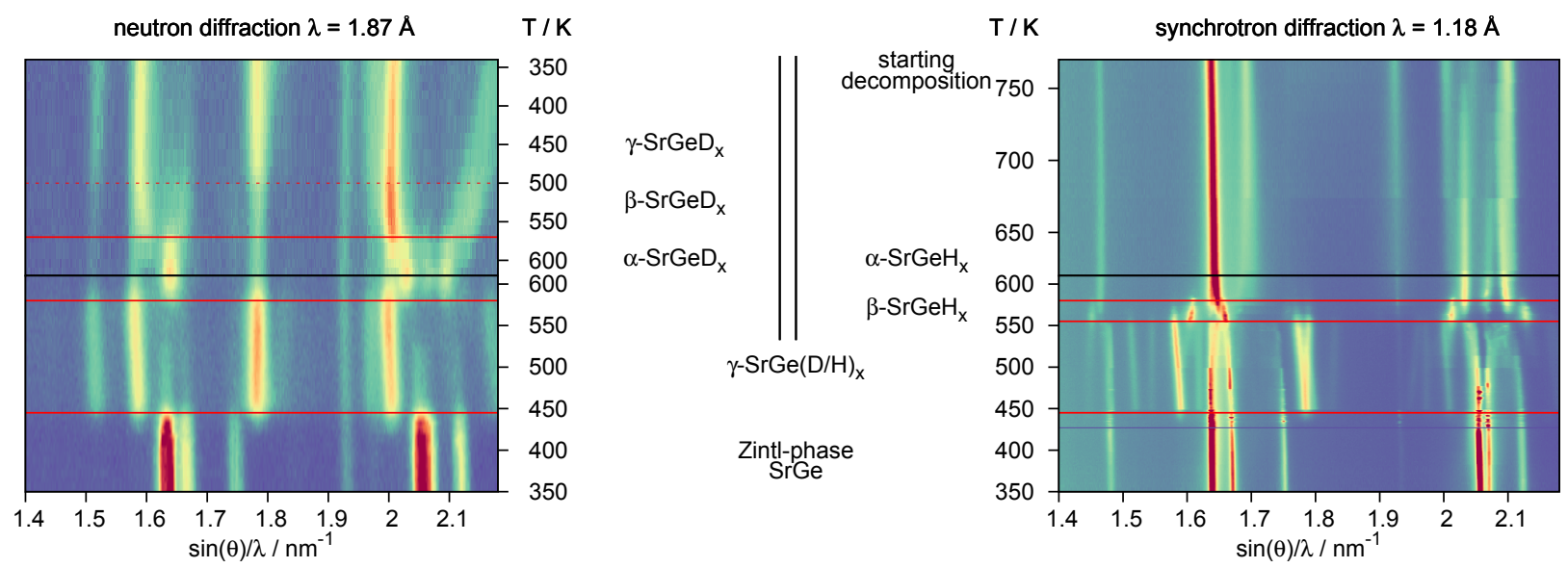

Figure 7: In situ powder neutron and synchrotron diffraction. Data were normalized to get a linear temperature scale. Both experiments were done at 5.0(1) $\mathrm{MPa}_{2}\left(\mathrm{D}_{2}\right)$ pressure. Synchrotron data were collected by $14 \mathrm{~s}$ exposure time and heating up to $770 \mathrm{~K}$ while using neutron diffraction the reversibility was probed heating to $623 \mathrm{~K}$ and cooling down afterwards.

pressure DSC can directly be correlated to in situ diffraction experiments. Fig. 7 shows in situ powder synchrotron and neutron diffraction at 5.0(1) $\mathrm{MPa}$ isobaric hydrogen (for synchrotron) or deuterium (for neutron diffraction) pressure. It can be clearly seen that the formation of $\gamma-\operatorname{SrGeD}_{\mathrm{y}}\left(\mathrm{H}_{\mathrm{y}}\right)$ is a one step reaction at $435 \mathrm{~K}$ which fits DSC data reasonably. Using synchrotron diffraction the first hydrogen release step can be monitored at $555 \mathrm{~K}$. Neutron diffraction cannot resolve this step, which could be caused by the small temperature window of this reaction and a stronger temperature inhomogeneity due to the large amount 
of sample. During the neutron diffraction experiment the reversibility was tested. The transition from $\alpha$ - to $\beta$-phase is reversible at $5 \mathrm{MPa} \mathrm{D}_{2}$ and approximately $575 \mathrm{~K}$. Due to the hysteresis during the reformation of $\gamma-\mathrm{SrGeD}_{\mathrm{y}}$ the $\beta$-phase can be resolved here. The decomposition into $\mathrm{SrGe}_{2}$ and $\mathrm{SrH}_{2}$ as seen in ex situ experiments is quite slow and could not be seen in the in situ synchrotron experiment.

\section{Structural changes during deuterium release and uptake}

The temperature dependent reaction of $\gamma$-SrGeD y under 5.0(1) MPa isobaric $\mathrm{D}_{2}$-pressure is discussed in more detail now. From the 2D-diffraction pattern it can be clearly seen that regions corresponding to the three distinct phases are present. Phase fractions and volume changes are shown in detail in Fig. S4.

$\gamma-\mathrm{SrGeD}_{\mathbf{y}}$ : During the heating ramp lattice parameter $a$ contracts while $b$ is increased. This reaches a maximum at $423 \mathrm{~K}$ (frame $60 /$ numor $926865^{23}$ ) and corresponds to a small deuterium uptake. The tetrahedral $\mathrm{Sr}_{4}$-voids are totally filled with deuterium within 3 e.s.u., thus the deuterium uptake corresponds to an increased occupation on site D4. This emphasizes the effect again, that $\gamma-\mathrm{SrGeD}_{\mathrm{y}}$ has a homogeneity range and releases some deuterium over time. After this maximum is reached, the lattice parameters show an inverse trend and the site corresponding to the Ge-D bond releases deuterium again. A minimal occupation of $48(4) \%$ at site D4 is refined before this phase collapses into $\alpha$-SrGeD . The intensity decrease of the (110) reflection while the (200) reflection stays constant is a clear indication of the deuterium loss as well (see Fig. S5). Within 3 e.s.u. the occupation of the tetrahedral voids does not change at all. The total breakup of the Ge-D bond during the formation of $\alpha-\mathrm{SrGeD}_{\mathrm{y}}$ at $575 \mathrm{~K}$ is close to the decomposition temperature of polymeric germane, $\left(\mathrm{GeH}_{\mathrm{y}}\right)_{\infty}$, which decomposes under $\mathrm{H}_{2}$ release at $470-520 \mathrm{~K} .{ }^{33}$

The Ge-Ge bond lengths depend on the changed deuterium occupation as well. While some of the Ge-D bonds break, the middle Ge3-Ge3 chain opens, thus reaching Ge-Ge 


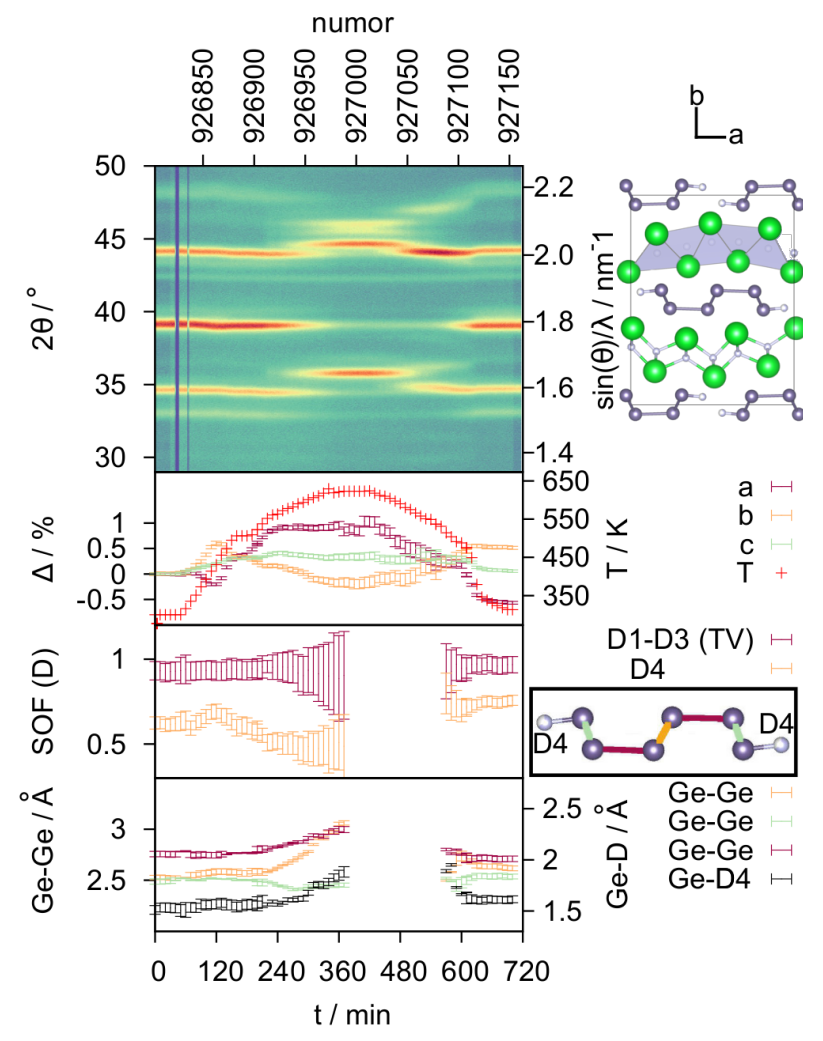

Figure 8: In situ PND data of $\gamma-\mathrm{SrGeD}_{\mathrm{y}}$ under 5.0(1) $\mathrm{MPa} \mathrm{D}_{2}$ pressure from $\mathrm{D} 20$ beamline, ILL $(\lambda=1.87 \AA) .{ }^{23}$ Error bars correspond to 3 e.s.u. as obtained by Rietveld refinement. SOF of the deuterium atoms in the tetrahedral voids (D1-D3(TV)) are averaged over all three positions. The different Ge-Ge bonds are drawn in the corresponding color (see inset box). 
distances close to the interchain distance Ge2-Ge3. During the deuterium release the Ge-D bond is slightly stretched as well.

Upon cooling $\gamma-\mathrm{SrGeD}_{\mathrm{y}}$ is reformed from $\beta-\mathrm{SrGeD}_{\mathrm{y}}$. At the end of the in situ experiment a final diffraction data set was measured at $313 \mathrm{~K}$. Lattice parameters $a$ and $b$ show a deviation of $\pm 0.5 \%$ compared to the starting material. This can be correlated to the increased deuterium content after the redeuteration. The average Ge-Ge bond is $2.627(8) \AA$, which is the same value as for the unhydrogenated Zintl phase SrGe. The terminal chain shows the shortest bond with $\mathrm{d}(\mathrm{Ge} 1-\mathrm{Ge} 2)=2.539(8) \AA$. The middle chain is $\mathrm{d}(\mathrm{Ge} 3-\mathrm{Ge} 3)=2.630(8) \AA$ and chain connecting bond is $\mathrm{d}(\mathrm{Ge} 2-\mathrm{Ge} 3)=2.712(9) \AA$. The Ge-D length reaches a slightly longer value than before with 1.609(11) $\AA$. The bond lengths in this freshly formed $\gamma$-SrGeD fit very well to the DFT calculations of a recently published study. ${ }^{15}$

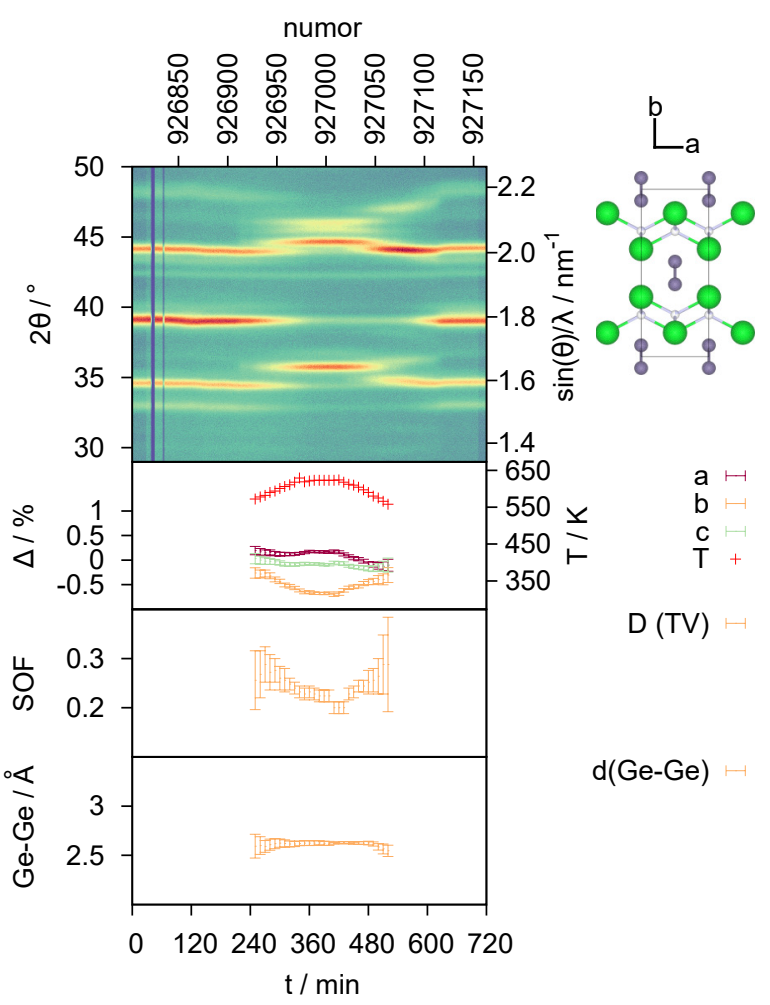

Figure 9: In situ PND data of the formation of $\alpha-\mathrm{SrGeD}_{\mathrm{y}}$ under 5.0(1) MPa $\mathrm{D}_{2}$ pressure from D20 beamline, ILL $(\lambda=1.87 \AA) .{ }^{23}$ Error bars correspond to 3 e.s.u. as obtained by Rietveld refinement. $\mathrm{D}(\mathrm{TV})=$ deuterium atom in tetrahedral $\mathrm{Sr}_{4}$ voids. 
$\alpha-\mathrm{SrGeD}_{\mathbf{y}}$ : Fig. 9 shows the evaluation of $\alpha-\mathrm{SrGeD}_{\mathrm{y}}$ during the in situ experiment. Upon heating lattice parameter $b$ contracts. At the maximum temperature of $623 \mathrm{~K}$ a minimal deuterium content was reached with 20.0(4)\% occupation of the tetrahedral $\mathrm{Sr}_{4}$-voids. When $\alpha-\mathrm{SrGeD}_{\mathrm{y}}$ reacts reversibly at $575 \mathrm{~K}$ a maximum deuterium content of $28.8(7) \%$ can be reached. The Ge-Ge chain does not change during this small deuterium release.

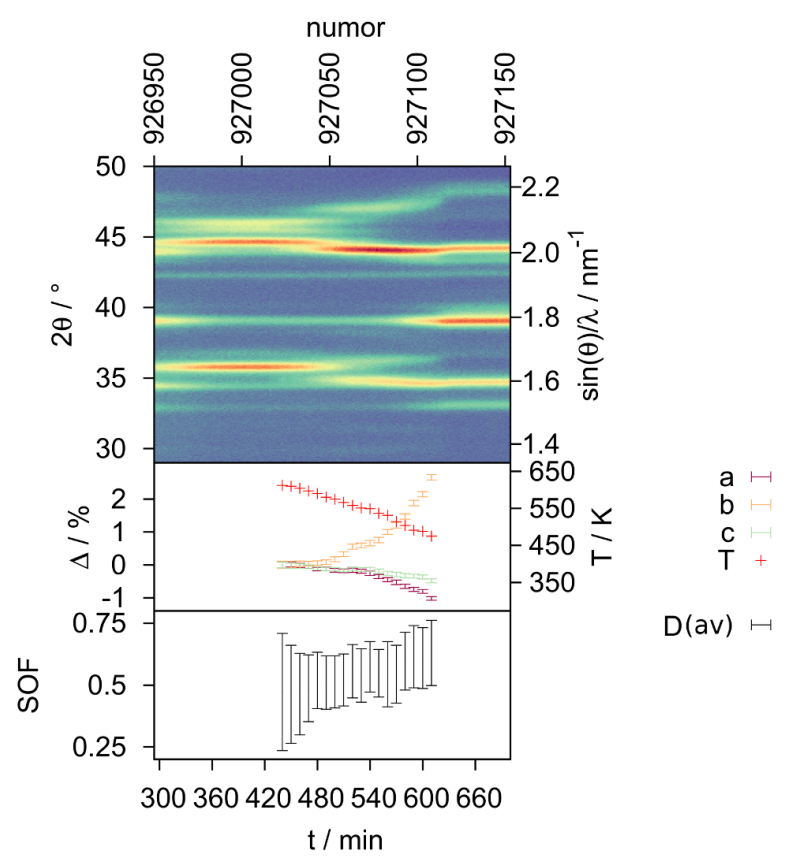

Figure 10: In situ PND data of the formation of $\beta-\mathrm{SrGeD}_{\mathrm{y}}$ under 5.0(1) MPa $\mathrm{D}_{2}$ pressure from D20 beamline, ILL $(\lambda=1.87 \AA) .{ }^{23}$ Error bars correspond to 3 e.s.u. as obtained by Rietveld refinement. Due to the averaged model only a limited number of parameters is evaluated. $\mathrm{D}(\mathrm{av})=\mathrm{SOF}$ averaged over all deuterium sites.

$\beta-\mathrm{SrGeD}_{\mathbf{y}}$ : The averaged model we suggest for $\beta-\mathrm{SrGeD}_{\mathrm{y}}$ allows some interpretation of deuterium occupations. The lattice parameters show the strongest effect within the $\mathrm{SrGe}-\mathrm{D}_{2}$ system. The $b$ lattice parameter strongly expands by $2.66(3) \%$ upon cooling from $613 \mathrm{~K}$ to $475 \mathrm{~K}$, while the overall deuterium content of the tetrahedral $\mathrm{Sr}_{4}$-voids increases from $47(8) \%$ to $63(4) \%$. Due to data quality a more thoroughly evaluation of in situ diffraction data on $\beta-\mathrm{SrGeD}_{\mathrm{y}}$ is still elusive. 


\section{Conclusion}

We recently reported about the existence of the hydrogen (deuterium) rich $\operatorname{SrGeD}_{4 / 3-\mathrm{x}}(\gamma-$ $\mathrm{SrGeD}_{\mathrm{y}}$ ) phase where the occurrence of a polyanionic hydride partial structure could be proven. ${ }^{15}$ Here, two additional phases in the $\mathrm{SrGe}-\mathrm{H}_{2} / \mathrm{D}_{2}$ system with lower hydrogen (deuterium) content are presented. From ex situ as well as in situ neutron diffraction data it was found that $\gamma-\mathrm{SrGeD}_{\mathrm{y}}$ has a homogeneity range. Tetrahedral $\mathrm{Sr}_{4}$-voids stay filled while the germanium bound D4-site looses hydrogen (deuterium). A maximal occupation of 68(1)\% for a freshly prepared sample was found. During the in situ experiment a minimal occupation of $48(4) \%$ was reached for the D4-site before the phase decomposed. Thus, the chemical formula can be established as $\mathrm{SrGeD}_{4 / 3-\mathrm{x}}$ with phase boundaries of at least $0.10<\mathrm{x}<0.17$.

From hydrogen pressure DSC we could show a pressure dependent decomposition above $540 \mathrm{~K}$ in several steps. In situ diffraction proved that the first step results from breaking of Ge-D bonds forming $\beta$ - $\mathrm{SrGeD}_{\mathrm{y}}$. The $\beta$-phase releases hydrogen (deuterium) reversibly to form $\alpha$-SrGeD y under $5 \mathrm{MPa} \mathrm{D}_{2}$ pressure at $575 \mathrm{~K}$. Upon cooling it takes up hydrogen (deuterium) and $\gamma$-SrGeD $\mathrm{y}$ is formed again below $520 \mathrm{~K}$. Within this hysteresis region $\beta$ $\mathrm{SrGeD}_{\mathrm{y}}$ shows a homogeneity range of at least $0.47<\mathrm{y}<0.63$ with the highest hydrogen (deuterium) content at the lowest temperature.

For $\alpha-\mathrm{SrGeD}_{\mathrm{y}}, \mathrm{y}=0.288(12)$ we find the highest hydrogen (deuterium) content at $575 \mathrm{~K}$ under $5 \mathrm{MPa}$ hydrogen (deuterium) pressure. Upon further heating the hydrogen (deuterium) content further decreases. The highest measured temperature at $5 \mathrm{MPa} \mathrm{D}_{2}$ was $623 \mathrm{~K}$ with $\mathrm{y}=0.200(4)$.

Ex situ experiments showed that under $5 \mathrm{MPa} \mathrm{H}_{2}$ pressure an irreversible reaction occurs at temperatures above $700 \mathrm{~K}$ forming $\mathrm{SrGe}_{2}$ and $\mathrm{SrH}_{2}$. Thus, hydrides of Zintl phases show a rich crystal chemistry with a variety of bonding situations and may be described with an extended Zintl-Klemm concept. As shown in this study, however, phase widths due to hydrogen vacancies may occur, which might be compensated by $\pi$-bonding within the polyanionic chains. It further demonstrates the potential of in situ studies, since both $\alpha$ - 
and $\beta$-phase in the SrGe- $\mathrm{D}_{2}$ system could only be found and studied this way.

\section{Dedication}

Dedicated to Prof. Lothar Beyer on the occasion of his 80th birthday.

\section{Supporting Information Available}

The following files are available free of charge. The supporting information file contains the following data: Tab. S1: Five sets of crystal structure data of $\gamma-\mathrm{SrGeD}_{\mathrm{y}}$; Fig. S1: Rietveld refinement of the crystal structure of $\gamma-\mathrm{SrGeD}_{\mathrm{y}}$ of 5 data sets; Fig. S2: Total angular range of in situ neutron diffraction of SrGe; Fig. S3: Total angular range of in situ neutron diffraction of $\gamma-\mathrm{SrGeD}_{\mathrm{y}}$; Fig. S4: Phase content and corresponing volumes of the different phases regarding to Fig. S3; Fig. S5: Low angle reflections regarding Fig. S3. CIF files for $\alpha$ - and $\beta-\mathrm{SrGeD}_{\mathrm{y}}$ are deposited as well.

\section{Acknowledgement}

This work has been supported by the Deutsche Forschungsgemeinschaft (DFG, grant Ko1803/81) and by Fonds der Chemischen Industry (FCI, grant 194371). The Institut Laue-Langevin (ILL) as well as Helmholtz-Zentrum Berlin (HZB, BER II and BESSY II) are acknowledged for providing beamtime. We thank Daniel Toebbens for general support at KMC-2 beamline, BESSY II and Alexandra Franz for support at E9 beamline, BER II. 


\section{References}

(1) Zintl, E.; Kaiser, H. Über die Fähigkeit der Elemente zur Bildung negativer Ionen. Z. Anorg. Allg. Chem. 1933, 211, 113-131.

(2) Zintl, E. Intermetallische Verbindungen. Angew. Chem. 1939, 52, 1-6.

(3) Schäfer, H.; Eisenmann, B.; Müller, W. Zintl-Phasen: Übergangsformen zwischen Metall- und Ionenbindung. Angew. Chem. 1973, 85, 742-760.

(4) Schäfer, H. On the Problem of Polar Intermetallic Compounds: The Stimulation of E. Zintl's Work for the Modern Chemistry of Intermetallics. Annu. Rev. Mater. Sci. 1985, $15,1-42$.

(5) Nesper, R. Chemische Bindungen - intermetallische Verbindungen. Angew. Chem. 1991, 103, 805-834.

(6) Corbett, J. D. Polyanionic Clusters and Networks of the Early p-Element Metals in the Solid State: Beyond the Zintl Boundary. Angew. Chem., Int. Ed. 2000, 39, 670-690.

(7) Nesper, R. The Zintl-Klemm Concept - A Historical Survey. Z. Anorg. Allg. Chem. 2014, 640, 2639-2648.

(8) Evers, J.; Weiss, A. Electrical properties of CrB type alkaline earth monosilicides and monogermanides. Solid State Commun. 1975, 17, 41 - 43.

(9) Reyes, E. C.; Nesper, R. Electronic Structure and Properties of the Alkaline Earth Monosilicides. J. Phys. Chem. C 2012, 116, 2536-2542.

(10) Kurylyshyn, I. M.; Fässler, T. F.; Fischer, A.; Hauf, C.; Eickerling, G.; Presnitz, M.; Scherer, W. Probing the Zintl-Klemm Concept: A Combined Experimental and Theoretical Charge Density Study of the Zintl Phase CaSi. Angew. Chem., Int. Ed. 2014, 53, 3029-3032. 
(11) Aoki, M.; Ohba, N.; Noritake, T.; Towata, S. Reversible hydriding and dehydriding properties of CaSi: Potential of metal silicides for hydrogen storage. Appl. Phys. Lett. 2004, 85, 387-388.

(12) Ohba, N.; Aoki, M.; Noritake, T.; Miwa, K.; Towata, S.-i. First-principles study of a hydrogen storage material CaSi. Phys. Rev. B 2005, 72, 075104.

(13) Wu, H.; Zhou, W.; Udovic, T. J.; Rush, J. J.; Yildirim, T. Structure and hydrogen bonding in $\mathrm{CaSiD}_{1+x}$ Issues about covalent bonding. Phys. Rev. B 2006, 74, 224101.

(14) Armbruster, M.; Wörle, M.; Krumeich, F.; Nesper, R. Structure and Properties of Hydrogenated Ca, Sr, Ba, and Eu Silicides. Z. Anorg. Allg. Chem. 2009, 635, 17581766.

(15) Auer, H.; Guehne, R.; Bertmer, M.; Weber, S.; Wenderoth, P.; Hansen, T.; Haase, J.; Kohlmann, H. Hydrides of Alkaline-earth-Tetrel (AeTt) Zintl phases: Covalent Tt-H Bonds from Silicon to Tin. submitted to Inorg. Chem 2016,

(16) Ångström, J.; Johansson, R.; Sarkar, T.; Sørby, M. H.; Zlotea, C.; Andersson, M. S.; Nordblad, P.; Scheicher, R. H.; Häussermann, U.; Sahlberg, M. Hydrogenation-Induced Structure and Property Changes in the Rare-Earth Metal Gallide NdGa: Evolution of a $[\mathrm{GaH}]^{2-}$ Polyanion Containing Peierls-like Ga-H Chains. Inorg. Chem. 2016, 55, $345-352$.

(17) Nedumkandathil, R.; Kranak, V. F.; Johansson, R.; Angström, J.; Balmes, O.; Andersson, M. S.; Nordblad, P.; Scheicher, R. H.; Sahlberg, M.; Häussermann, U. Hydrogenation Induced Structure and Property Changes in GdGa. J. Solid State Chem. 2016, 239, $184-191$.

(18) Møller, K. T.; Hansen, B. R. S.; Dippel, A.-C.; Jørgensen, J.-E.; Jensen, T. R. Characterization of Gas-Solid Reactions using In Situ Powder X-ray Diffraction. Z. Anorg. Allg. Chem. 2014, 640, 3029-3043. 
(19) Hansen, T. C.; Kohlmann, H. Chemical Reactions followed by in situ Neutron Powder Diffraction. Z. Anorg. Allg. Chem. 2014, 640, 3044-3063.

(20) Westlake, D.; Shaked, H.; Mason, P.; McCart, B.; Mueller, M.; Matsumoto, T.; Amano, M. International Symposium on the Properties and Applications of Metal Hydrides Interstitial site occupation in ZrNiH. J. Less-Common Met. 1982, 88, 17 -23 .

(21) Hansen, T. C.; Henry, P. F.; Fischer, H. E.; Torregrossa, J.; Convert, P. The D20 instrument at the ILL: a versatile high-intensity two-axis neutron diffractometer. Meas. Sci. and Technol. 2008, 19, 034001.

(22) Kohlmann, H.; Kurtzemann, N.; Weihrich, R.; Hansen, T. In situ Neutron Powder Diffraction on Intermediate Hydrides of $\mathrm{MgPd}_{3}$ in a Novel Sapphire Gas Pressure Cell. Z. Anorg. Allg. Chem. 2009, 635, 2399-2405.

(23) Kohlmann, H.; Auer, H.; Götze, A.; Hansen, T.; Weber, S. Reaction pathways to the Zintl phase hydrides CaSiH and $\mathrm{MGeH}_{\mathrm{x}}(\mathrm{M}=\mathrm{Sr}$, Ba). 2015; doi 10.5291/ILL-DATA.522-734.

(24) Mayer, H.; Knorr, K.; Többens, D.; Stüßer, N.; Lampert, G. E9: The New HighResolution Neutron Powder Diffractometer at the Berlin Neutron Scattering Center. European Powder Diffraction EPDIC 7. 2001; pp 288-293.

(25) Többens, D. M.; Zander, S. KMC-2: an X-ray beamline with dedicated diffraction and XAS endstations at BESSY II. Journal of large-scale research facilities 2016, 2, A49.

(26) Rodríguez-Carvajal, J. Recent advances in magnetic structure determination by neutron powder diffraction. Phys. B 1993, 192, 55 - 69 .

(27) Rodríguez-Carvajal, J. FullProf.2k, Version 5.30 - Mar2012-ILL JRC. 2012. 
(28) Momma, K.; Izumi, F. VESTA3 for three-dimensional visualization of crystal, volumetric and morphology data. J. Appl. Crystallogr. 2011, 44, 1272-1276.

(29) VESTA - Visualisation for Electronic and STructural Analysis, version 3.3.1.

(30) Gelato, L. M.; Parthé, E. STRUCTURE TIDY - a computer program to standardize crystal structure data. J. Appl. Crystallogr. 1987, 20, 139-143.

(31) Häussermann, U.; Kranak, V. F.; Puhakainen, K. In Zintl Phases; Fässler, T. F., Ed.; Structure and Bonding; Springer Berlin Heidelberg, 2011; Vol. 139; pp 143-161.

(32) Reyes, E. C.; Stalder, E. D.; Mensing, C.; Budnyk, S.; Nesper, R. Unexpected Magnetism in Alkaline Earth Monosilicides. The Journal of Physical Chemistry C 2011, $115,1090-1095$.

(33) Bianco, E.; Butler, S.; Jiang, S.; Restrepo, O. D.; Windl, W.; Goldberger, J. E. Stability and Exfoliation of Germanane: A Germanium Graphane Analogue. ACS Nano 2013, 7, 4414-4421. 


\section{For Table of Contents Only}

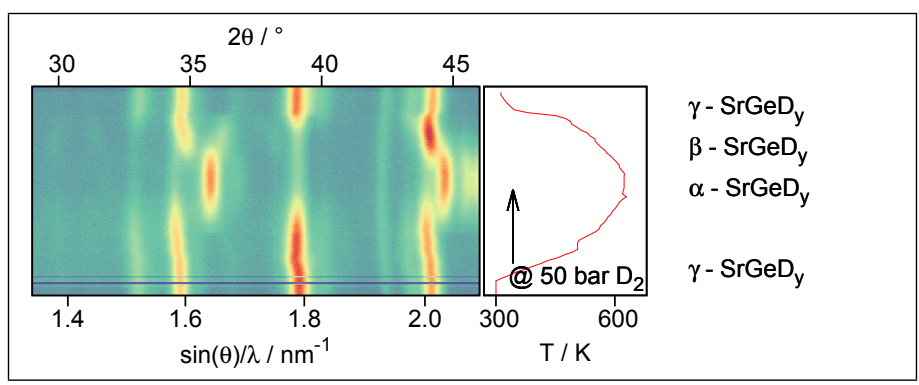

The in situ reaction of $\gamma-\mathrm{SrGeD}_{\mathrm{y}}\left(\mathrm{SrGeD}_{4 / 3-\mathrm{x}}\right)$ under 50 bar $\mathrm{D}_{2}$ pressure leads to a reversible formation of two deuterium poorer Zintl phase deuterides. 